\title{
Vaccinations in rheumatic diseases
}

\author{
Martyna Szwejkowska ${ }^{1}$, Ernest Kuchar ${ }^{1,2}$ \\ ${ }^{1}$ Department of Pediatrics with Clinical Assessment Unit, Medical University of Warsaw, Poland \\ ${ }^{2}$ Polish Society of Vaccinology
}

Vaccines are products of biological origin, which, by inducing immunity, prevent or alleviate infections and infectious diseases. The risk of infection is particularly high in patients with autoimmune inflammatory rheumatic diseases (AIIRD) because associated diseases and immunosuppressive therapies increase the contagious burden. The European League Against Rheumatism (EULAR) helps us in planning vaccinations for this group of patients, and at the end of 2019 updated their recommendations. EULAR addressed them to all doctors involved in the care of patients with AllRD. The 2019 update for vaccination in adult patients with AIIRD is divided into six overarching principles and nine recommendations. According to the opinion of Polish experts, immunization in children with rheumatic diseases can follow the same recommendations [1].

Based on the EULAR guideline, the rheumatological team should assess patients with AIIRD every year for vaccination status and indications for their update or continuation. Such an individualized immunization program should be carefully explained to the patient and implemented in cooperation with a primary care physician and a rheumatological team [2]. The best approach is to administer vaccines during "steady-state", a remission of AIIRD before planned immunosuppression (especially before therapy reducing B cell counts). For this patient group, we should plan vaccinations at least six months after and four weeks before the next treatment cycle. In cases where this time interval is not possible, immunization may be considered as part of the B-cell reduction therapy, taking into account the potential non-optimal response to the vaccine. Limited knowledge about the safety and immunogenicity of vaccines during active disease creates a contraindication. It is worth emphasizing that, in severe cases, we should not delay the necessary vaccinations [2].

Patients with AlIRD on glucocorticosteroids (GC) or disease-modifying antirheumatic drugs (DMARDs) can safely receive inactivated, "killed" vaccines. Data from clinical studies confirmed that administration of the vaccines against influenza, pneumococci, tetanus toxoid, hepatitis B (HBV), hepatitis A (HAV), and human papillomavirus (HPV) is effective and safe among those patients [1, 2]. The administration of live, attenuated vaccines during immunosuppression should be avoided in patients with AIIRD because live attenuated microorganisms can potentially cause infection. However, there is a possibility of careful use of the measles, mumps, and rubella (MMR), and herpes zoster vaccines. Based on their safety data, they can be considered in people with AIIRD with a low degree of immunosuppression and a high chance of contracting measles (travelers) or herpes zoster (risk groups) [2].

Due to extensive evidence of safety and good immunogenicity, influenza, and pneumococcal vaccination, we should think about them in most patients with rheumatic diseases [2]. These people, especially immunosuppressive patients, have a higher risk of getting sick compared to the general population $[3,4]$. When we plan to vaccinate people with AIIRD, we should remember that rituximab has a strong effect on $B$ cells. Therefore, when we are planning any of the above vaccinations, they should be implemented before rituximab treatment. Patients with AllRD should receive a tetanus toxoid vaccine as recommended for the general population. However, passive immunization with tetanus immunoglobulins (for example in the case of wound management) is the preferred method of tetanus prophylaxis in patients treated with rituximab [1].

The HAV and HBV vaccines should only be given to patients at risk. These include seronegative patients who travel or are residents in endemic countries and persons at increased risk of exposure to HBV (for example, medical personnel, home contact persons, sexual partners of persons with chronic HBV infection, intravenous drug users). CDC recommends passive immunization or booster vaccination in patients not vaccinated or with an insufficient response to hepatitis B [5]. Patients

Address for correspondence:

Ernest Kuchar, Department of Pediatrics with Clinical Assessment Unit, Pediatric Hospital, Medical University of Warsaw,

63A Żwirki i Wigury St., 02-091 Warsaw, Poland, e-mail: ernest.kuchar@gmail.com

Submitted: 18.04.2020; Accepted: 22.04.2020 
with AlIRD are at increased risk for herpes zoster $(\mathrm{HZ})$ compared to the general population. Chickenpox evaluation should be considered before administration of a live $\mathrm{HZ}$ vaccine to prevent primary infection. The safety and efficacy of the inactivated $\mathrm{HZ}$ vaccine have not yet been studied in patients with AlIRD, but it seems to be an attractive alternative to live immunization [6].

Patients with AIIRD during immunosuppression should avoid yellow fever vaccination because of the risk of producing an infection [7]. Patients with AIIRD, in particular patients with systemic lupus erythematosus (SLE), should receive vaccination against human papillomavirus (HPV) as recommended for the general population, because most of the evidence regarding HPV epidemiology in patients with rheumatic diseases is based on studies in women with SLE [8]. According to the Infectious Diseases Society of America, household members of people with AIIRD should be vaccinated according to national guidelines [9]. Newborn babies of mothers who took biological medicines at the end of the second and third trimesters of pregnancy should not receive live vaccines for the first six months of life [10]. Measuring the level of a given biological drug in the serum can help make a vaccination decision with live vaccines.

The rheumatological team in cooperation with primary care physicians should educate patients about the indications and contraindications for specific vaccinations and inform them about the risk-benefit ratio. Further research is needed to make the preparation of personalized recommendations easier to protect the patient with AIIRD.

In guidance on routine immunization services during the COVID-19 pandemic in the WHO European Region of 20 March 2020, the World Health Organization (WHO) issued recommendations on the implementation of vaccination during the COVID-19 pandemic in the European Region [11]. The WHO points out that immunization services are an essential component of health care and should be maintained as long as COVID-19 response measures allow. The WHO has prioritized vaccination against pneumococci and seasonal influenza for vulnerable groups. As a result, on 17 April 2020, the Ministry of Health and GIS published a communication on the resumption of immunizations in Poland, in which it recommends the universalization of pneumococcal and seasonal influenza vaccination in adult risk groups, including people over 60 years of age and the chronically ill, as they are at high risk of pneumonia.

The authors declare no conflict of interest.

\section{References}

1. Jóźwiak H, Wysocki J. Szczepienia ochronne w profilaktyce chorób zakaźnych. Przewodnik Lekarza/Guide for GPs 2001; 4: 42-47.

2. Furer V, Rondaan C, Heijstek MW, et al. 2019 update of EULAR recommendations for vaccination in adult patients with autoimmune inflammatory rheumatic diseases. Ann Rheum Dis 2020; 79: 39-52, DOI: 10.1136/annrheumdis-2019-215882.

3. Dirven L, Huizinga TWJ, Allaart CF. Risk factors for reported influenza and influenza-like symptoms in patients with rheumatoid arthritis. Scand J Rheumatol 2012; 41: 359-36, DOI: 10.3109/03009742.2012.670729.

4. Shea KM, Edelsberg J, Weycker, et al. Rates of pneumococcal disease in adults with chronic medical conditions. Open Forum Infect Dis 2014;1: ofu024, DOI: 10.1093/ofid/ofu024.

5. https://www.cdc.gov/vaccines/pubs/pinkbook/downloads/ hepb.pdf [Accessed 20.04.2020].

6. Lal H, Cunningham AL, Godeaux O, et al. Efficacy of an adjuvanted herpes zoster subunit vaccine in older adults. N Engl J Med 2015; 372: 2087-2896, DOI: 10.1056/NEJMoa1501184.

7. Staples JE, Gershman M, Fischer M, et al. Yellow fever vaccine: recommendations of the Advisory Committee on immunization practices (ACIP). MMWR Recomm Rep 2010; 59: 1-27.

8. Soybilgic A, Onel KB, Utset T, et al. Safety and immunogenicity of the quadrivalent HPV vaccine in female Systemic Lupus Erythematosus patients aged 12 to 26 years. Pediatr Rheumatol Online J 2013; 11: 29, DOI: 10.1186/1546-0096-11-29.

9. Rubin LG, Levin MJ, Ljungman P, et al. 2013 IDSA clinical practice guideline for vaccination of the immunocompromised host. Clin Infect Dis 2014; 58: 309-318, DOI: 10.1093/cid/cit816.

10. Götestam Skorpen C, Hoeltzenbein M, Tincani A, et al. The EULAR points to consider for use of antirheumatic drugs before pregnancy, and during pregnancy and lactation. Ann Rheum Dis 2016; 75: 795-810, DOI: 10.1136/annrheumdis-2015-208840.

11. http://www.euro.who.int/en/health-topics/health-emergencies/ coronavirus-covid-19/novel-coronavirus-2019-ncov-technicalguidance/coronavirus-disease-covid-19-outbreak-technicalguidance-europe/guidance-on-routine-immunization-servicesduring-covid-19-pandemic-in-the-who-european-region-2020 [Accessed 20.04.2020]. 